\title{
Non-Linear Field Theories II. Canonical Equations and Quantization*
}

\author{
Peter G. Bergmann and Johanna H. M. Brunings \\ Department of Physics, Syracuse University, Syracuse, New York
}

\begin{abstract}
In this paper, a covariant field theory of the general type of the theory of relativity is brought into the canonical form and then quantized. Particles are assumed to be represented as singularities of the field. Primarily, we had to overcome two difficulties. First, the variational integral should be extended only over that spacetime domain which is free of singularities. Since the location of the singular world lines cannot be known until after the integration of the field equations has been completed, we have introduced a second set of coordinates-called "parameters" in this paperwhich will serve as variables of integration and in terms of which the motions of the particles can be arbitrarily prescribed. The second difficulty arises in that the expressions for the canonical momentum densities cannot be solved with respect to the partial time derivatives of the field variables; this circumstance precludes the construction of the Hamiltonian by the usual methods. Nevertheless, we have shown that a Hamiltonian exists, though it is not uniquely determined by the Lagrangian; our Hamiltonian contains an arbitrary linear combination of the eight algebraic relationships that exist between the canonical variables at each world point. The canonical field equations have the usual form. They are covariant if the choice of Hamiltonian is left open. The
\end{abstract}

eight algebraic constraints on the canonical variables at each point are all integrals of the field equations. So are the Poisson brackets between the canonical variables (at the same time). When this system of equations and constraints is quantized, the property of general covariance can be used to carry out a proof of the covariance of the whole theory, including the commutation relations, that requires none of the computational effort usually required in theories that are merely Lorentz invariant. Once the system of equations has been. completed, it turns out that the covariance goes much farther than was required originally. Because of the introduction of the parameters, the ordinary coordinates of space-time turn formally, at least, into dynamical variables, and the usual canonical transformations, with respect to which the theory is covariant, transform the coordinates, the original field variables, and the canonical conjugates of both together. The canonical conjugates of the coordinates are the expressions ordinarily interpreted as energy and momentum densities. The physical significance of these canonical transformations, which cause the world points to lose their identities, is not yet understood.

\section{INTRODUCTION AND REVIEW}

$I^{\mathrm{N}}$ a previous paper, ${ }^{1}$ attention was called to the special physical features of completely covariant field theories. If the field equations are the Euler-Lagrange equations of a four-dimensional variational principle, it is possible to obtain equations of motion for particles by considering the particles as singular time-like curves in space-time and by requiring that outside these singularities the field equations are satisfied everywhere. This dependence of the equations of motion on the field equations was first established for the general theory of relativity by A. Einstein and his co-workers. ${ }^{2-4}$

In $\mathrm{I}$, it was assumed that the field equations can be derived from a variational principle which contains only the field variables and their first partial derivatives, but no higher derivatives, and which is so constructed that the resulting Euler-Lagrange equations are covariant. It was shown that the field equations satisfy identities similar to the Bianchi identities of the general theory of relativity, that they cannot be solved with respect to the highest second derivatives with respect to some one coordinate, and that the continuation of solutions which are given on an initial space-like hyper-

\footnotetext{
* This work was supported in part by the Office of Naval Research under Contract N6-onr-248.

1 P. G. Bergmann, Phys. Rev. 75, 680 (1949). Referred to in this paper as I. Reference to formulas in that paper is made as (I-4.2)

${ }^{2}$ A. Einstein, L. Infeld, and B. Hoffmann, Ann. Math. 39, 65 (1938).

${ }^{3}$ A. Einstein and L. Infeld, Ann. Math. 41, 455 (1940).

${ }^{4}$ L. Infeld and P: R. Wallace, Phys. Rev, 57, 797 (1940).
} etc. surface in the direction of time is not uniquely determined by the equations. It was further shown that in this generalized theory (in which the nature of the field remains for the time being unspecified) the equations of motion of field singularities are determined by the field equations outside in the same manner as in the general theory of relativity.

Because the motion of field singularities is determined by the field outside the singularities (if each singularity is enclosed in a small three-dimensional spherical surface) the field equations need to be satisfied only on and outside that spherical envelope. The classical (i.e., unquantized) theory can be carried through without considering the self-energy of the particles or any other divergent quantities. To this extent, this type of theory is the most nearly self-consistent classical field theory yet devised. It still falls short of the "perfect" field theory in that it fails to represent particles as nonsingular solutions of the field equations. The purpose of our present program is to attempt the quantization of such a field theory and to see to what extent the usual divergences of quantum field theories can be avoided. The present paper is a step in this program. We shall first of all indicate how a variational principle can be set up which requires integration only over the nonsingular domain of space-time. We shall then develop the canonical form of the classical theory, and we shall show finally that if the classical field variables are replaced by operators, then the usual commutation relations are covariant.

For the convenience of the reader, we shall collect 
here those results and formulas of $\mathrm{I}$ which are needed in this paper. We denote the field variables by $y_{A}(A=1$, $\cdots, N)$, and their derivatives with respect to one of the four coordinates $x^{\rho}$ by $y_{A, \rho}$. We shall assume that the Lagrangian $L$ depends only on $y_{A}$ and on $y_{A, \rho}$. The field equations then take the form

$$
0=\partial^{A} L-\left(\partial^{A \rho} L\right)_{, \rho} \equiv L^{A}
$$

where the symbol $\partial^{A}$ denotes differentiation of a function of the field variables with respect to $y_{A}$ and the symbol $\partial^{A \rho}$ differentiation with respect to $y_{A, \rho}$. An infinitesimal coordinate transformation is defined by means of the four functions $\xi^{\rho}$, which represent the (infinitesimal) changes in the values of the coordinates of a fixed world point with the original coordinates $x^{\rho}$. It is assumed that the transformation law of the field variables with respect to infinitesimal coordinate transformations possesses the form

$$
\bar{\delta} y_{A}=F_{A \mu}{ }^{B \nu} \xi^{\mu},{ }_{\nu} y_{B}-y_{A, \mu} \xi^{\mu} .
$$

The coefficients $F_{A \mu}^{B \nu}$ are a set of constants characteristic for the field variables. The last term in (I-2.3) is a "transport term," which must be inserted if the value of $y_{A}$ at a point with the original coordinates $\mathbf{x}$ is to be compared with the transformed $y_{A}$ at the point which after the transformation possesses the coordinates $\mathbf{x}$ (and, therefore, originally had the coordinates $x-\xi)$.

The transformation matrix $F_{A \mu}{ }^{B \nu}$ must satisfy certain commutation relations if the infinitesimal transformation law is to generate a finite transformation law.

$$
F_{A \mu}{ }^{C \nu} F_{C_{\rho}}{ }^{B \sigma}-F_{A \rho}{ }^{C \sigma} F_{C_{\mu}}{ }^{B \nu} \equiv \delta_{\rho}{ }^{\nu} F_{A \mu}{ }^{B \sigma}-\delta_{\mu}{ }^{\sigma} F_{A_{\rho}}{ }^{B \nu} \text {. }
$$

The Lagrangian from which the field equations are to be derived is assumed to satisfy a transformation law of the form

$$
\bar{\delta} L=Q^{\rho}, \rho
$$

which assures that the left-hand sides of the field equations transform covariantly according to the formula

$$
\bar{\delta} L^{B}=-F_{A \mu}{ }^{B \nu} \xi^{\mu},{ }_{\nu} L^{A}-\left(L^{B} \xi^{\mu}\right), \mu .
$$

It can easily be shown that the $Q^{\rho}$ of (I-2.5) are the following four expressions:

$$
Q^{\rho} \equiv F_{A \mu}^{B \rho} y_{B} L^{A} \xi^{\mu}+\partial^{A \rho} L \bar{\delta} y_{A} .
$$

The most important property of the field equations for what follows is that they satisfy four differential identities: When we substitute for $L^{A}$ the full expressions in terms of the field variables and their first and second derivatives, the four equations

$$
F_{A \mu}{ }^{B \rho}\left(L^{A} y_{B}\right)_{, \rho}+L^{A} y_{A, \mu} \equiv 0
$$

are identically satisfied. These equations contain the third derivatives of the $y_{A}$ linearly. Their coefficients must vanish identically by themselves. These conditions turn out to be

$$
\begin{array}{r}
\left(F_{A \mu}{ }^{B \rho} L^{A C \sigma \tau}+F_{A \mu}^{B \sigma} L^{A C \tau \rho}+F_{A \mu}^{B \tau} L^{A C \rho \sigma}\right) y_{B} \equiv 0, \\
L^{A C \rho \sigma}=-\frac{1}{2}\left(\partial^{A \rho} \partial^{C \sigma} L+\partial^{A \sigma} \partial^{C \rho} L\right) .
\end{array}
$$

Finally, if the field equations are satisfied, then the divergence of certain expressions vanishes, which are usually identified with the energy momentum densities and energy-momentum fluxes:

$$
\begin{aligned}
t_{\iota}{ }^{\rho},{ }_{\rho} & =0 \\
t_{\iota}{ }^{\kappa} & =\delta_{\iota}{ }{ } L-y_{A,}{ }_{\iota} \partial^{A}{ }^{\kappa} L .
\end{aligned}
$$

In these formulas and all that follow, the summation convention for dummy indices is used for all types of indices without distinction.

\section{THE PARAMETER FORMALISM}

In his formulation of the equations of motion in the general theory of relativity, Einstein worked exclusively with the field equations and with the identities that exist between them without taking recourse to the Lagrangian. In attempting to construct a Hamiltonian as a preliminary to quantization, we shall need to refer to the original variational principle. The volume integral in space-time $\int L \mathbf{d x}$ that is to be made stationary with respect to variations of the field variables in the interior of the domain of integration can be extended over any four-dimensional domain desired, and the field equations will then be satisfied throughout the interior of that domain. Naturally, the domain of integration should not include regions where the equations cannot be satisfied, and it should in particular exclude the world lines along which particles move.

In this connection, there arises a peculiar difficulty. Because the motions of the particles are determined by the field equations outside, we cannot predict the location of the singular world lines until after we have accomplished the integration of the field equations. On the other hand, it will, of course, be necessary to choose the domain of integration for the Lagrangian even before the field equations are formulated, let alone solved. This difficulty can be resolved if we introduce, in addition to the coordinates $x^{\rho}$, a second set of coordinates that can serve as variables of integration. Call the second set $u^{s}(s=1, \cdots, 3), t$. To avoid any ambiguities of language, this second set will be referred to as "parameters," and the one parameter $t$ will occasionally be called "time." The transformation law leading from the coordinates to the parameters shall not be specified initially, except that it shall be assumed that the Jacobian of the transformation,

$$
J \equiv\left|\frac{\partial\left(x^{\rho}\right)}{\partial\left(u^{s}, t\right)}\right|,
$$

shall not vanish: In terms of parameters, the basic variational principle goes over into

$$
\begin{aligned}
\delta S & =0 \\
S & =\int_{t} \int_{\mathbf{u}} J L \mathbf{d} \mathbf{u} d t .
\end{aligned}
$$


The coordinates $x^{\rho}$ are considered as field variables, along with the $y_{A}$. Naturally, the additional field equations will not lead to a determination of the $x^{p}$ as functions of the parameters if the field equations are to be satisfied in a singly connected four-dimensional domain. Straightforward computation shows that the EulerLagrange equations belonging to the new field variables $x$ reduce to (I-3.8) and are, thus, contained in the original field equations. In the presence of line singularities, however, the coordinates of the singularities are, in fact, determined by certain integrals over the (space-like, two-dimensional) closed surfaces that enclose the singularities and which are derivable from the divergence relations (I-3.8) or from the stronger relations (I-3.11), which hold even inside the singular regions and which go over into (I-3.8) on the outside.

Nothing prevents us from prescribing the motions of the singularities in terms of the parameters initially and from extending the integral (2.2) over the multiply connected domain of the four parameters in which we propose to satisfy the field equations. If the integral is made stationary with respect to variations of the field variables $y_{A}, x^{\boldsymbol{p}}$ in the interior of that domain, both the field equations and the equations of motion will be satisfied.

The formal device just described permits us to formulate our variational principle so that the integrand of $S$ is everywhere finite. Naturally, the field equations must be covariant both with respect to coordinate transformations and with respect to parameter transformations. With respect to the latter, the field variables are scalars and their first derivatives covariant vectors. In what follows, all $(N+4)$ field variables will be accepted as such.

Since partial derivatives will occasionally have to be taken, both with respect to $x^{p}$ and with respect to $u^{s}$ and $t$, we shall uniformly denote differentiation with respect to $t$ by the dot symbol and differentiation with respect to $u^{s}$ by the symbol ${ }_{\mid s}$. We have, thus, for example,

and

$$
y_{A, \rho}=\dot{y}_{A} t_{, \rho}+y_{A \mid s} u_{, \rho}^{s}
$$

$$
\begin{gathered}
\dot{y}_{A}=y_{A, \rho} \dot{x}^{\rho} \\
y_{A \mid s}=y_{A, \rho} x_{\mid s} .
\end{gathered}
$$

\section{CONONICAL VARIABLES AND THE HAMILTONIAN}

Into the formalism just described canonical momentum densities can be introduced in the usual manner by means of the defining equations,

$$
\pi^{A} \equiv \partial^{A \cdot}(L J)=J t, \partial^{A \rho} L
$$

and

$$
\begin{aligned}
\lambda_{\rho} \equiv \partial(L J) / \partial \dot{x}^{\rho} & =J t_{, \sigma}\left(L \delta_{\rho}{ }^{\sigma}-y^{A},{ }_{\rho} \partial^{A \sigma} L\right) \\
& \equiv J t_{,} t_{\rho}{ }^{\sigma} .
\end{aligned}
$$

$t_{\rho}{ }^{\sigma}$ has been defined by means of Eq. (I-3.8). These $(N+4)$ canonical momentum densities satisfy a number of algebraic relations. With the help of Eq. (I-3.6), four of these relations can be obtained. By multiplying
(I-3.6) by $J^{2} \cdot t, \rho t, t_{, \tau}$ one obtains, after a few obvious calculations,

$$
\partial^{A \cdot}\left(F_{B \mu}^{C \tau} y_{C} \pi^{B} J t, \tau\right)=0 .
$$

The expression within the parentheses of (3.3) could depend on $\dot{x}^{\prime}$ either directly or through the $y_{A, \rho}$. The latter possibility is excluded because

$$
\left.\begin{array}{rl}
\left(\partial y^{A}, \sigma / \partial \dot{x}^{\rho}\right) \partial^{A \sigma} & =-y_{A, \rho} t_{,} \partial^{A \sigma} \\
& =-y_{A, \rho} \partial^{A^{\bullet}}
\end{array}\right\} .
$$

But the same expression in (3.3) cannot depends on $\dot{x}^{\rho}$ directly, either, because

$$
\frac{\partial(J t, \sigma)}{\partial \dot{x}^{\rho}}=0 .
$$

It follows that integration of the four Eqs. (3.3) leads to the four equations

$$
F_{A \mu}^{B \tau} y_{B} \pi^{A} J t_{, \tau}=K_{\mu}\left(y_{C}, y_{C \mid s}, x_{\mid s}\right),
$$

where the functions on the right hand side are determined in any actual theory and, in any case, do not contain either $\dot{y}_{A}$ or $\dot{x}^{\rho}$ as arguments.

Additional algebraic relationships can be obtained directly from the defining Eqs. (3.1), (3.2). By multiplying the obvious identity

$$
\lambda_{\rho}-J t,{ }_{\rho} L+y_{A, \rho} \pi^{A}=0
$$

by $x^{p}{ }_{1 s}$, we find

$$
x_{\mid s}^{\rho} \lambda_{\rho}+y_{A \mid s} \pi^{A}=0,
$$

three algebraic relationships between the canonical variables which are free of "time" derivatives. If we multiply. Eq. (3.7) by $\dot{x}^{p}$, we get

$$
J L \equiv \dot{y}_{A} \pi^{A}+\dot{x}^{\rho} \lambda_{\rho}
$$

showing formally that the Lagrangian $J L$ is homogeneous of the first degree in the arguments $\dot{y}_{A}, \dot{x}^{p}$. If the Lagrangian $J L$ is of the first degree, then the derivatives $\pi^{A}$ and $\lambda_{\rho}$ are homogeneous of the zeroth degree. It is well known that $n$ zeroth-degree functions of $n$ arguments cannot be algebraically independent of each other. It is possible to obtain a differential relationship for this algebraic condition. Let the condition be

$$
g\left(\pi^{A}, \lambda_{\rho}, y_{A}, y_{A \mid s}, x_{\mid s}^{\rho}\right) \equiv 0
$$

to be satisfied identically if the momenta are replaced by their defining equations. Differentiation leads to

$$
\begin{aligned}
0= & \frac{\partial g}{\partial \pi^{A}} \delta \pi^{A}+\frac{\partial g}{\partial \lambda_{\rho}} \delta \lambda_{\rho}+\frac{\delta g}{\delta y_{A}} \delta y_{A}+\frac{\delta g}{\delta x^{\rho}} \delta x^{\rho} \\
& +\left(\frac{\partial g}{\partial y_{A \mid s}} \delta y_{A}+\frac{\partial g}{\partial x_{\mid s}^{\rho}} \delta x^{\rho}\right)=\left[\frac{\partial g}{\partial \pi^{B}} \partial^{A \cdot} \partial^{B} \cdot(L J)\right. \\
& \left.+\frac{\partial g}{\partial \lambda_{\sigma}} \partial^{A} \cdot \partial_{\sigma} \cdot(L J)\right] \delta \dot{y}_{A}+\left[\frac{\partial g}{\partial \pi^{B}} \partial_{\rho} \cdot \partial^{B \cdot}(L J)\right.
\end{aligned}
$$




$$
\begin{gathered}
\left.+\frac{\partial g}{\partial \lambda_{\sigma}} \partial_{\rho} . \partial_{\sigma} .(L J)\right] \delta \dot{x}_{\rho}+\left[\frac{\partial g}{\partial \pi^{B}} \frac{\partial \pi^{B}}{\partial y_{A}}+\frac{\partial g}{\partial \lambda_{\sigma}} \frac{\partial \lambda_{\sigma}}{\partial y_{A}}\right. \\
\left.-\left(\frac{\partial g}{\partial \pi^{B}} \frac{\partial \pi^{B}}{\partial y_{A \mid s}}+\frac{\partial g}{\partial \lambda_{\sigma}} \frac{\partial \lambda_{\sigma}}{\partial y_{A \mid s}}\right)_{\mid s}+\frac{\delta g}{\delta y_{A}}\right] \delta y_{A} \\
-\left(\frac{\partial g}{\partial \pi^{B}} \frac{\partial \pi^{B}}{\partial x_{\mid s}^{\rho}}+\frac{\partial g}{\partial \lambda_{\sigma}} \frac{\partial \lambda_{\sigma}}{\partial x_{\mid s}^{\rho_{1 s}}}+\frac{\partial g}{\partial x_{\mid s}^{\rho}}\right)_{\mid s} \delta x^{\rho} \\
+\left[\left(\frac{\partial g}{\partial y_{A \mid s}}+\frac{\partial g}{\partial \pi^{B}} \frac{\partial \pi^{B}}{\partial y_{A \mid s}}+\frac{\partial g}{\partial \lambda_{\sigma}} \frac{\partial \lambda_{\sigma}}{\partial y_{A \mid s}}\right) \delta y_{A}\right. \\
\left.+\left(\frac{\partial g}{\partial \cdot x_{\mid s}^{\rho}}+\frac{\partial g}{\partial \pi^{B}} \frac{\partial \pi^{B}}{\partial x_{\mid s}^{\rho}}+\frac{\partial g}{\partial \lambda_{\sigma}} \frac{\partial \lambda_{\sigma}}{\partial x_{\mid s}^{\rho}}\right) \delta x^{\rho}\right]_{\mid s}
\end{gathered}
$$

If we integrate this differential form over a space-like domain (with $t$ constant) and if we consider the $\delta y_{A}$, $\delta \dot{y}_{A}$, etc., as arbitrary variations of the independent field variables, we get the conditions

$$
\begin{aligned}
& \frac{\partial g}{\partial \pi^{B}} \partial^{A \cdot} \cdot \partial^{B \cdot}(J L)+\frac{\partial g}{\partial \lambda_{\sigma}} \partial^{A} \cdot \partial_{\sigma} \cdot(J L)=0 \\
& \frac{\partial g}{\partial \pi^{B}} \partial_{\rho} \cdot \partial^{B \cdot}(J L)+\frac{\partial g}{\partial \lambda_{\sigma}} \partial_{\rho} \cdot \partial_{\sigma} \cdot(J L)=0 \\
& \frac{\partial g}{\partial y_{A}}+\frac{\partial g}{\partial \pi^{B}} \frac{\partial \pi^{B}}{\partial y_{A}}+\frac{\partial g}{\partial \lambda_{\sigma}} \frac{\partial \lambda_{\sigma}}{\partial y_{A}}=0 \\
& \frac{\partial g}{\partial y_{A \mid s}}+\frac{\partial g}{\partial \pi^{B}} \frac{\partial \pi^{B}}{\partial y_{A \mid s}}+\frac{\partial g}{\partial \lambda_{\sigma}} \frac{\partial \lambda_{\sigma}}{\partial y_{A \mid s}}=0 \\
& \frac{\partial g}{\partial x_{\mid s}^{\rho}}+\frac{\partial g}{\partial \pi^{B}} \frac{\partial \pi^{B}}{\partial x_{\mid s}^{\beta}}+\frac{\partial g}{\partial \lambda_{\sigma}} \frac{\partial \lambda_{\sigma}}{\partial x_{\mid s}^{\rho}}=0 .
\end{aligned}
$$

Equations (3.12) are, of course, satisfied by any one of the seven algebraic "constraints" on the canonical momenta (3.6), (3.8) as well as by the eighth relationship yet to be obtained. The first two Eqs. (3.12) can be understood in the sense that the $(N+4)$ quantities $\left(\partial g / \partial \pi^{A}\right),\left(\partial g / \partial \lambda_{\rho}\right)$ form a "null vector" of the (singular) matrix

$$
\Lambda=\left(\begin{array}{ll}
\partial^{A \cdot} \partial^{B \cdot}(J L), & \partial^{A} \cdot \partial_{\sigma} \cdot(J L) \\
\partial_{\rho} \cdot \partial^{B} \cdot(J L), & \partial_{\rho} \cdot \partial_{\sigma} \cdot(J L)
\end{array}\right) .
$$

This matrix has eight linearly independent null vectors. Seven of these null vectors will lead back to the relationships already obtained. An eighth null vector possesses the components $\dot{y}_{A}, \dot{x}^{p}$. The expressions

$$
\left.\begin{array}{c}
\dot{y}_{B} \partial^{B \cdot} \cdot \partial^{A} \cdot(J L)+\dot{x}^{\sigma} \partial_{\sigma} \cdot \partial^{A} \cdot(J L)=0 \\
\dot{y}_{B} \partial^{B \cdot} \partial_{\rho} \cdot(J L)+\dot{x}^{\sigma} \partial_{\sigma} \cdot \partial_{\rho} \cdot(J L)=0
\end{array}\right\}
$$

vanish, because of the homogeneity property of the
Lagrangian $J L$. We set, therefore,

$$
\begin{aligned}
& \frac{\partial g}{\partial \pi^{A}}=\dot{y}_{A}, \quad \frac{\partial g}{\partial \lambda_{\rho}}=\dot{x}^{\rho}, \\
& \frac{\delta g}{\delta y_{A}}=-\left(\dot{y}_{B} \frac{\partial \pi^{B}}{\partial y_{A}}+\dot{x}^{\sigma} \frac{\partial \lambda_{\sigma}}{\partial y_{A}}\right) \\
& \quad+\left(\dot{y}_{B} \frac{\partial \pi^{B}}{\partial y_{A \mid s}}+\dot{x}^{\sigma} \frac{\partial \lambda_{\sigma}}{\partial y_{A \mid s}}\right)_{\mid s} \equiv-\frac{\delta(J L)}{\delta y_{A}}, \\
& \frac{\delta g}{\delta x^{\rho}}=-\frac{\delta(J L)}{\delta x^{\rho}} .
\end{aligned}
$$

If the field Eqs. (I-2.2) are satisfied, the partial (or rather variational) derivatives of the Lagrangian with respect to $y_{A}$ and $x^{\rho}$ satisfy the equations

$$
\left.\begin{array}{l}
\frac{\delta(J L)}{\delta y_{A}}=\dot{\pi}^{A} \\
\frac{\delta(J L)}{\delta x^{\rho}}=\dot{\lambda}_{\rho}
\end{array}\right\}
$$

As a result, we have

$\dot{\pi}^{A}=-\frac{\delta g}{\delta y_{A}}, \quad \dot{\lambda}_{\rho}=-\frac{\delta g}{\delta x^{\rho}}, \quad \dot{y}_{A}=+\frac{\partial g}{\partial \pi^{A}}, \quad \dot{x}^{\rho}=+\frac{\partial g}{\partial \lambda_{\rho}}$,

the canonical equations, with the function $g$ as the Hamiltonian density.

The function $g$ is not completely determined by the Eqs. (3.15). Suppose we have obtained an algebraic constraint $g$ which is a result of the homogeneity of the right hand sides of Eqs. (3.1), (3.2) with respect to the $\dot{y}_{A}, \dot{x}^{\rho}$. Then first of all, this constraint can be multiplied by any non-vanishing function $\nu_{0}$ of the $x$ without destroying the validity of the Eqs. (3.15). All we do by such a change in $g$ is to choose a new parameter system, $\left(u^{s}, t^{*}\right)$, in which $t^{*}$ is a function of the parameters $\left(u^{s}, t\right)$ of the original system. Furthermore, if we add to some chosen $g$ a linear combination of the remaining seven constraints, the coefficients being arbitrary functions of the $\mathbf{u}, t$, we shall obtain a new set of canonical equations which differs from the original set in that the coordinates and parameters occurring in the new set are related to the coordinates and parameters of the original set of canonical equations by specific coordinate and parameter transformations.

We shall call a particular choice of the function $g$ the Hamiltonian density and designate that choice by $H$. Once $H$ has been chosen, the "equations of motion" (in the sense in which this term is used in quantum mechanical literature) are no longer covariant. Covariance can be maintained only as long as we are willing to reserve the choice of $H$. 
In addition to the Hamiltonian density $H$, we shall introduce the Hamiltonian itself.

$$
\mathfrak{H}=\int_{\mathfrak{u}} H d u^{1} d u^{2} d u^{3}
$$

$\mathfrak{F C}$ is a functional and depends on the values of all the canonical variables throughout a whole space-like threedimensional surface $t=$ constant. "Functional derivatives" of a functional with respect to its arguments are to be understood in accordance with the defining equation

$$
\begin{aligned}
& \delta \mathfrak{F}=\int_{\mathbf{u}}\left[\frac{\delta \mathcal{F}}{\delta y_{A}(\mathbf{u})} \delta y_{A}(\mathbf{u})+\frac{\delta \mathcal{F}}{\delta \pi^{A}(\mathbf{u})} \delta \pi^{A}(\mathbf{u})\right.
\end{aligned}
$$

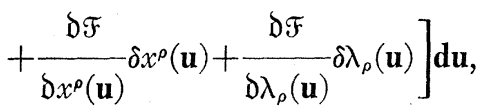

where $\mathcal{F}$ is any functional which depends on the canonical variables and their space derivatives throughout a three-dimensional surface $t=$ constant. For an integral like the Hamiltonian, the functional derivatives reduce to the variational derivatives of the integrand,

$$
\frac{\delta \mathcal{H C}}{\delta y_{A}(\mathbf{u})}=\left.\frac{\delta H}{\delta y_{A}}\right|_{\mathbf{u}}, \text { etc. }
$$

With the help of the Hamiltonian $\mathcal{H}$ and its functional derivatives, the canonical equations can be rewritten in the form

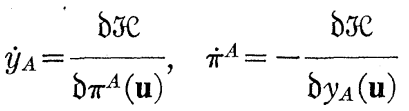

$$
\begin{aligned}
& \dot{x}^{\rho}=\frac{\delta \mathcal{H C}}{\delta \lambda_{\rho}(\mathbf{u})}, \quad \dot{\lambda}_{\rho}=-\frac{\delta \mathcal{H C}}{\delta x^{\rho}(\mathbf{u})} .
\end{aligned}
$$

If we introduce a functional $\mathfrak{F}$ which depends on the canonical variables on one surface $t=$ constant, then we can summarize Eqs. (3.21) in the form

$$
\dot{\mathfrak{F}}=(\mathfrak{F}, \mathfrak{F})
$$

where the right hand side, the "Poisson bracket" between $\mathcal{F}$ and $\mathcal{H C}$, stands for

$$
\begin{aligned}
& (\mathfrak{F}, \mathfrak{H C}) \equiv \int_{\mathbf{u}}\left\{\frac{\mathfrak{D F}}{\mathfrak{D} y_{A}(\mathbf{u})} \frac{\mathfrak{D} \mathcal{H}}{\mathfrak{D} \pi^{A}(\mathbf{u})}-\frac{\mathfrak{D} F}{\mathfrak{d} \pi^{A}(\mathbf{u})} \frac{\mathfrak{D} \mathcal{H}}{\mathfrak{D} y_{A}(\mathbf{u})}\right.
\end{aligned}
$$

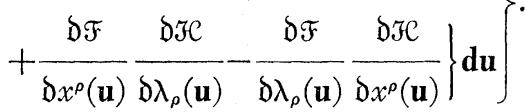

It should be noted that not every functional of the original field variables $y_{A}$ and their first derivatives $y_{A, \rho}$ can be converted into a functional of the canonical variables. If $\Psi$ is a functional of $y_{A}, \dot{y}_{A}, x^{\rho}, \dot{x}^{\rho}$, then it can be represented as a functional of the canonical variables only if

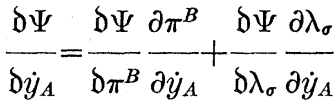

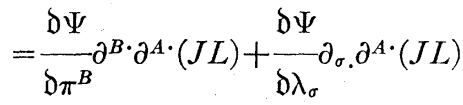

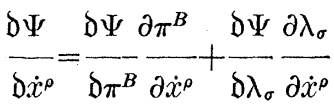

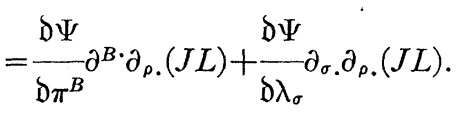

In other words, the "dot products" of the "vector" $\left(\mathfrak{D} \Psi / \mathfrak{D} \dot{y}_{A}, \mathfrak{D} \Psi / \mathfrak{D} \dot{x}^{\rho}\right)$ by any one of the eight null vectors of the matrix $\Lambda$, Eq. (3.13), must vanish. With 9 some linear functional of the eight algebraic constraints on the canonical variables, Eq. (3.24) can be rewritten in the form

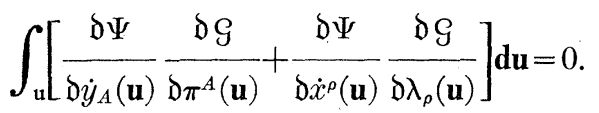

Functionals which satisfy the condition (3.25) for all constraints and which can, therefore, be considered to depend only on the canonical variables shall be called "dynamical variables.",

The functionals $\mathcal{G}$ themselves are not only dynamical variables, they are "integrals of the motion": If the constraints are satisfied on one surface $t=$ constant, then they remain satisfied for other values of $t$, provided the Eqs. (3.21) or (3.22) are satisfied. The proof follows immediately if the functional $\mathcal{G}$ is substituted in the expression for the Poisson bracket (3.23) and if the expressions for $\mathfrak{D} \mathcal{G} / \mathfrak{D} y_{A}$ and for $\mathfrak{D} \mathcal{G} / \mathfrak{D} x^{\rho}$ are substituted from Eqs. (3.12).

\section{POISSON BRACKETS AND CANONICAL TRANSFORMATIONS}

It is appropriate in the canonical formalism to consider functionals and their time dependence in preference to functions. Most of the calculations become simplified if they are carried out on functionals, if we are only willing to assume that the two dimensional surface integrals which appear in integrations by parts give zero contributions. Ordinary functions may be considered as functionals whose functional derivatives with respect to canonical variables at points other than the one specified space point vanish.

As a preparation for the quantization of the theory, we shall in this section define the canonical transformations so that with respect to them the Poisson brackets of two dynamical variables are invariant, and we shall show that the canonical equations generate a canonical transformation. 
Since we shall treat from now on the $y_{A}$ and $x^{p}$ uniformly, and likewise the $(N+4)$ canonically conjugate momentum densities, all formulas will be simplified if we combine the $y_{A}$ and $x^{p}$ into $y_{a}, a=1, \cdots, N+4$, and likewise the $\pi^{A}$ and $\lambda_{\rho}$ into $\pi^{a}$. Small Latin indices from the first half of the alphabet will now always run from 1 to $N+4$. With this notation, the Poisson bracket between two arbitrary dynamical variables $\mathcal{E}$ and $\mathfrak{F}$ is defined as

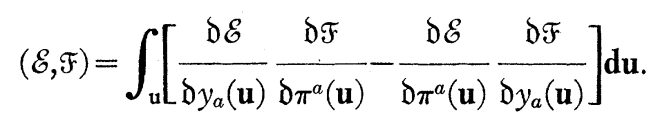

Our dynamical system is completely defined by the requirements that for all values of $t$

$$
\dot{F}=(\mathfrak{F}, \mathfrak{H})+\frac{\partial \mathfrak{F}}{\partial t}
$$

and that for $t=t_{0}$ the constraints

$$
\mathcal{S}=0 \text {. }
$$

Now we can introduce a new system of canonical variables $y_{a}^{\prime}\left(\mathbf{u}^{\prime}\right), \pi^{a^{\prime}}\left(\mathbf{u}^{\prime}\right)$ in such a manner that the system of Eqs. (4.2) goes over into a new system possessing the same form. In the usual manner, we shall introduce a "generating functional."

and set

$$
\mathfrak{C}=\mathfrak{C}\left(y_{a}(\mathbf{u}), \pi^{a^{\prime}}\left(\mathbf{u}^{\prime}\right), t\right)
$$

$$
\begin{aligned}
& \pi^{a}(\mathbf{u})=\frac{\mathfrak{D C}}{\mathfrak{D} y_{a}(\mathbf{u})} \\
& \left.y_{a}^{\prime}\left(\mathbf{u}^{\prime}\right)=\frac{\delta \mathfrak{C}}{\delta \pi^{a^{\prime}}\left(\mathbf{u}^{\prime}\right)}\right\} \text {. } \\
& \mathfrak{H}^{\prime}=\mathfrak{H}+\frac{\partial \mathcal{C}}{\partial t}
\end{aligned}
$$

The identical transformation is generated by the functional

$$
\left.\begin{array}{rl}
\mathcal{C}_{(E)} & =\int_{\mathbf{u}} \int_{\mathbf{u}^{\prime}} y_{a}(\mathbf{u}) \pi^{b^{\prime}}\left(\mathbf{u}^{\prime}\right) \delta_{b}{ }^{a} \delta\left(\mathbf{u}^{\prime}-\mathbf{u}\right) \mathbf{d} \mathbf{u}^{\prime} \mathbf{d} \mathbf{u} \\
& =\int_{\mathbf{u}} y_{a}(\mathbf{u}) \pi^{a^{\prime}}(\mathbf{u}) \mathbf{d} \mathbf{u}
\end{array}\right\}
$$

The infinitesimal transformation belonging to the transformation (4.4), (4.5) is, therefore,

$$
\left.\begin{array}{l}
\mathcal{C}=\mathcal{C}_{(E)}+c\left(y_{a}(\mathbf{u}), \pi^{a^{\prime}}(\mathbf{u}), t\right), \\
\delta y_{a}=\frac{\delta c}{\delta \pi^{a}}, \quad \delta \pi^{a}=-\frac{\delta c}{\delta y_{a}}, \quad \delta \mathcal{H C}=\frac{\partial c}{\partial t}
\end{array}\right\} .
$$

Now it is easy to prove that with respect to the in- finitesimal transformation the Poisson brackets do not change. We have

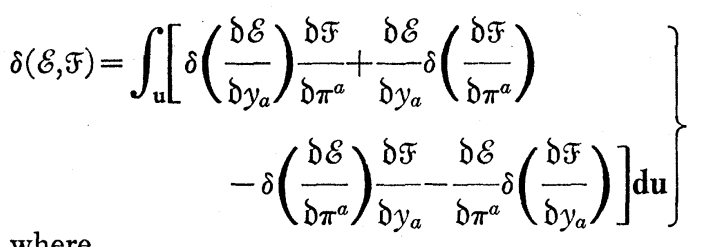

where

$$
\begin{aligned}
& \delta\left[\frac{\delta \mathcal{E}}{\delta y_{a}(\mathbf{u})}\right]=-\int_{\mathbf{u}^{\prime}}\left[\frac{\delta \mathcal{E}}{\delta y_{b}\left(\mathbf{u}^{\prime}\right)} \frac{\delta \delta y_{b}\left(\mathbf{u}^{\prime}\right)}{\delta y_{a}(\mathbf{u})}\right.
\end{aligned}
$$

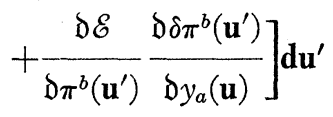

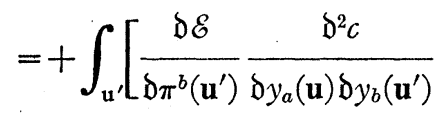

$$
\begin{aligned}
& \left.-\frac{\delta \mathcal{E}}{\delta y_{b}\left(\mathbf{u}^{\prime}\right)} \frac{\grave{D}^{2} c}{\delta y_{a}(\mathbf{u}) \delta \pi^{b}\left(\mathbf{u}^{\prime}\right)}\right] \mathbf{d \mathbf { u } ^ { \prime }} \text { etc. }
\end{aligned}
$$

If the expressions (4.9) are substituted into Eq. (4.8), the right-hand side vanishes identically. Since the result obtained for the infinitesimal transformation can be extended immediately to the finite transformation, it follows that the Poisson brackets are invariant with respect to the canonical transformations (4.4), (4.5).

Returning to the "equations of motion," (4.2), we shall now show that the right-hand side is invariant with respect to an infinitesimal canonical transformation. Because of the invariance of the bracket operation as such, the transformation of Eq. (4.2) yields

$$
\left.\begin{array}{l}
\delta \dot{F}=(\mathfrak{F , \delta \mathcal { H }})+\delta\left(\frac{\partial \mathscr{F}}{\partial t}\right) \\
\delta \mathcal{H C} \equiv \frac{\partial c}{\partial t}
\end{array}\right\} .
$$

The last term is necessary because of the assumed explicit time dependence of the transformation. It equals

$$
\begin{aligned}
& \delta\left(\frac{\partial \mathcal{F}}{\partial t}\right)=-\int_{\mathbf{u}}\left[\frac{\partial \mathcal{F}}{\delta y_{a}(\mathbf{u})} \frac{\partial \delta y_{a}(\mathbf{u})}{\partial t}\right.
\end{aligned}
$$

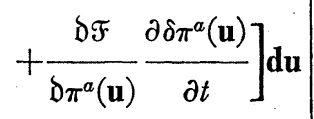

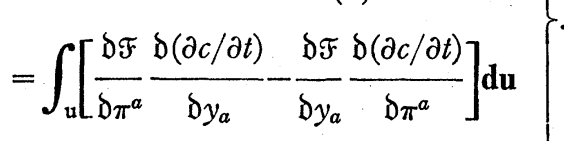

$$
\begin{aligned}
& =-\left(F, \frac{\partial c}{\partial t}\right) \text {. }
\end{aligned}
$$

As a result, the right-hand side of (4.10) vanishes. 
If the "time" derivatives of all dynamical variables are invariant with respect to canonical transformations, it follows that the constraints $\mathcal{G}$ remain integrals of the motion. Setting them equal to zero initially assures their being zero permanently. Herewith, the proof of the invariance of the system of canonical equations (4.2), (4.3) with respect to canonical transformations (4.4), (4.5) is completed.

It is very easy to show that the change of the canonical variables with time is nothing but a special canonical transformation. Comparison of Eq. (4.7) with (3.21) shows that the generating functional is the Hamiltonian itself. Because the equations which connect the values of the canonical coordinates at two different times $t_{1}$ and $t_{2}$ with each other are equivalent to a canonical transformation, it follows that if $\mathcal{E}$ and $\mathcal{F}$ are two functionals whose values are independent of $t$ (though they will in general depend explicitly on the canonical variables and on $t$ ), then their Poisson bracket $(\mathcal{E}, \mathcal{F})$ is again constant in time.

\section{QUANTIZATION}

In this section, we shall demonstrate the covariance of the quantized theory which is obtained from the "classical" (i.e., unquantized) theory in the usual manner.

As is customary, we shall consider the canonical variables and also all the dynamical variables (in accordance with the defining Eq. (3.24)) as Hermitian operators acting on a "wave function" $\Psi$. For any two Hermitian operators $\mathcal{E}$ and $\mathcal{F}$ which are the analogues of dynamical variables in the classical theory we shall require that the commutator,

$$
[\mathcal{E}, \mathfrak{F}] \equiv-\frac{i}{\hbar}-(\mathcal{E F}-\mathscr{F} \mathcal{E})
$$

be the operator that is the analogue to the Poisson bracket $(\mathcal{E}, \mathcal{F})$. This correspondence requirement can always be satisfied if the operators $\mathcal{E}$ and $\mathcal{F}$ are built up from the canonical operators in the same manner as the analogous dynamical variables from the canonical variables. The sequence of canonical operators in products has to be arranged so that the resulting operator is Hermitian.

The dependence of the operators on $t$ is given by the new equation of motion

$$
\mathfrak{F}=[\mathfrak{F ,} \mathfrak{F}]+\frac{\partial \mathfrak{F}}{\partial t}
$$

and the commutation relations between the canonical variables at the time $t$ are given by the requirement (5.1),

$$
\begin{gathered}
{\left[y_{a}(\mathbf{u}), y_{b}\left(\mathbf{u}^{\prime}\right)\right]=0 \quad\left[\pi^{a}(\mathbf{u}), \pi^{b}\left(\mathbf{u}^{\prime}\right)\right]=0} \\
{\left[y_{a}(\mathbf{u}), \pi^{b}\left(\mathbf{u}^{\prime}\right)\right]=\delta_{a}^{b} \delta\left(\mathbf{u}-\mathbf{u}^{\prime}\right) .}
\end{gathered}
$$

Integration of the equations of motion gives for any operator which does not depend on $t$ explicitly the expression

$$
\left.\mathscr{F}\right|_{t}=\left.\exp \left(\frac{i}{\hbar} \int_{t_{0}}^{t} \mathscr{F}(\tau) d \tau\right) \mathscr{F}\right|_{t_{0}} \exp \left(-\frac{i}{\hbar} \int_{t_{0}}^{t} \mathscr{F}(\tau) d \tau\right)
$$

All the operators $\&$ commute with the Hamiltonian. We shall require that at some initial time $t_{0}$ every $\mathcal{G}$ satisfies the condition

$$
g \Psi=0 .
$$

That condition will then be satisfied permanently.

The equations of motion (5.2), the commutation relations (5.1) or (5.3), and the constraints (5.5) are together a complete (Heisenberg) representation of a quantized theory. We have now to show that this theory is covariant with respect to coordinate and with respect to parameter transformations. That covariance is, of course, contingent on the continued free choice of the Hamiltonian as an arbitrary linear combination of constraint conditions $\mathcal{G}$, provided only that the coefficient of the eighth condition, the one that expresses the homogeneity of the Lagrangian, is nowhere zero in the $(\mathbf{u}, t)$ continuum. Transition to a new coordinate system or to a new parameter system will carry the equations of motion over into themselves, but in general the form of the Hamiltonian will change. The problem is whether the commutation relations and the constraints will be reproduced by a transformation.

To answer this question requires no computational work, because of the special structure of our theory. It turns out that the constraint conditions as well as the commutation relations are covariant simply because they are integrals of the motion. Consider a solution of the equations of motion which also satisfies the constraints and the commutation relations. Naturally, this solution is available in a particular coordinate system and in a particular parameter system. Now it is possible to envisage a particular point of the $(\mathbf{u}, t)$ continuum and to inquire how the constraints as well as the commutation relations will be affected by a coordinate or by a parameter transformation. Such a transformation can be achieved solely by an integration of the equations of motion. We merely need to carry our solution in one direction of $t$ for a finite distance and then to integrate it backward, but with a different choice of Hamiltonian. This switch in the Hamiltonian will result in our returning to the original space-time point with a different set of coordinates and parameters. Specifically, that addition of a functional that is linear in the constraints (3.6) will transform the coordinates, while the constraints (3.8) and the eighth constraint are related to $\mathbf{u}$-transformations and $t$-transformations, respectively. Since integration of the equations of motion with arbitrary $\mathfrak{H C}$ preserves both the form of the constraints and the form of the commutation relations, covariance of all these relationships is assured. 


\section{DISCUSSION}

With this paper, we have succeeded in putting a covariant theory into such a form that its equations can be integrated even in the presence of (world line) singularities. Moreover, we have shown that a Hamiltonian exists even though the differential equations do not determine uniquely the solutions of the standard Cauchy problem. This lack of determinancy of the solutions is reflected in a partial freedom of choice for the Hamiltonian: It is permissible to add to a given Hamiltonian an arbitrary linear combination of the eight algebraic constraints that hold between the canonical variables at each world point, with the only restriction that in the resulting new Hamiltonian the coefficient of the eighth constraint must not vanish. ${ }^{* *}$ Adoption of a particular linear combination of the constraints as the Hamiltonian amounts to the choice of a particular set of coordinate-parameter conditions. No matter what the choice of Hamiltonian, all other constraints $\mathcal{G}$ as well as the commutators of the canonical variables commute with the Hamiltonian and are, therefore, constant in time.

Our parameter formalism has formal similarity with one developed by P. Weiss. ${ }^{5}$ It differs in that Weiss uses his analogue primarily to vary the domain of integration of the variational principle. His surfaces $t=$ constant are necessarily closed, while ours are not only open, but, in the presence of particles, multiply

\footnotetext{
** If this coefficient were permitted to vanish, the resulting choice of the parameter system would establish a linear dependence between $d u^{s}$ and $d t$.

5 P. Weiss, Proc. Roy. Soc. A156, 192 (1936); A169, 102, 119 (1938).
}

connected. Also, Weiss has apparently taken the emergence of the $x^{p}$ as dynamical variables not as seriously as we do.

Our formalism is covariant not only with respect to coordinate and parameter transformations, but also with respect to the much more general group of the canonical transformations (4.4), (4.5). Once the coordinates $x^{\rho}$ and the energy-momentum densities $\lambda_{\rho}$ are included in the dynamical variables, the canonical transformations tend to break down the differences in character between the quantized field variables and the classical space-time continuum. In contrast to Snyder's assumptions, ${ }^{6}$ our coordinates commute with each other, but not with the energy-momentum densities. The dynamical character of any particle coordinates follows automatically, but probably does not exhaust the physical significance of the coordinate commutation relations.

Throughout the mathematical discussion of the earlier sections it has been assumed implicitly that the matrix $\Lambda$, (3.13), has only eight null vectors. This assumption will not hold if the theory is covariant with respect to further transformation groups. Such a group will be the gauge transformation group if the theory is to contain an electromagnetic field with a conservation law of charge. In the event of any such additional covariance properties, the number of algebraic constraints will be increased correspondingly. They may be added to the Hamiltonian, and they will, in any case, commute with the Hamiltonian. The basic structure of the theory is not affected by such additional constraints.

\footnotetext{
${ }^{6}$ H. Snyder, Phys. Rev. 71, 38 (1947); 72, 68 (1947).
} 